\title{
Equipos multiprofesionales-multidisciplinares en el ámbito educativo - legislación comparada y regulación de políticas educacionales en Argentina y Brasil ${ }^{\star}$
}

Equipes multiprofissionais-multidisciplinares no ambiente educacional - legislação comparada e regulação de políticas educacionais na Argentina e no Brasil

\section{Multiprofessional-multidisciplinary teams in the educational environment - comparative legislation and regulation of educational policies in Argentina and Brazil}

\author{
Silvia Cristina Yannoulas** \\ Mónica Girolami***
}

\begin{abstract}
DOI: $10.1590 / 0104-4060.51392$
* Este texto fue elaborado en el contexto del proyecto de investigación "El trabajo de los equipos multidisciplinares en las escuelas y en la Política Educacional" del Grupo de Investigación TEDis - Trabajo, Educación y Discriminación, de la Universidad de Brasília - UnB (www.tedis. unb.br); y cuenta con el apoyo del CNPq (beca de Iniciación Científica, beca de productividad en investigación y apoyo a proyectos en el área de las Ciencias Sociales y Humanas). Las ideas centrales fueron traducidas y adaptadas para fines pedagógicos (YANNOULAS; LENARDUZZI, 2016), e incluidas en el texto generador del Taller "Entre la Cooperación y el Conflicto: Equipos Multiprofesionales-Multidisciplinares en el Ambiente Educacional", realizado en 2016, en la Universidad Nacional de Entre Ríos (Paraná, Argentina). Agradecemos las contribuciones a los participantes del mencionado taller, las cuales permitieron enriquecer la versión final del texto.

${ }^{* *}$ Universidad de Brasília. Departamento de Servicio Social. Brasília, Distrito Federal, Brasil. Campus Universitário Darcy Ribeiro. Ala Norte. CEP: 70910-900. E-mail: silviayannoulas@unb.br.

*** Universidad de Buenos Aires. Buenos Aires, Argentina. Santiago del Estero 1029, C1075AAU CABA, Argentina. E-mail: monicagirolami@gmail.com.
\end{abstract}




\section{RESUMEN}

Las demandas al servicio/trabajo social por parte de las instituciones educacionales en Argentina y Brasil están asociadas al proceso histórico de establecimiento de la obligatoriedad escolar y la universalización del derecho a la educación. Esa obligatoriedad fue legislada por primera vez en 1884 en la Argentina a través de la Ley n ${ }^{\circ} 1.420$ y en la Constitución Federal de 1934 en el Brasil, donde encontró mayores dificultades para ser institucionalizada debido a la histórica fuerza descentralizadora ${ }^{1}$. A pesar de ello y con base en la revisión de literatura realizada, se puede observar que en ambos países hubo una serie de etapas más o menos definidas en la relación entre la obligatoriedad escolar, la universalización de la educación básica, la situación de pobreza del alumnado y la institucionalización de servicios/ trabajos sociales en el sistema educativo: etapa higienista, desarrollista, autoritaria y democratizadora/neoliberal.

Palabras clave: Derecho a la educación. Equipos multiprofesionales-multidisciplinares. Intersetorialidad. Legislación educativa. Procesos de trabajo escolar-educacional.

\section{RESUMO}

As demandas ao serviço/trabalho social realizadas pelas instituições educacionais na Argentina e no Brasil estão associadas ao processo histórico de estabelecimento da obrigatoriedade escolar e à universalização do direito à educação. Essa obrigatoriedade foi legislada pela primeira vez na Argentina, através da Lei n ${ }^{\circ} 1.420$, em 1884, e no Brasil, através da Constituição Federal, em 1934, donde encontrou maiores dificuldades para ser institucionalizada, devido à histórica força descentralizadora deste país. Apesar disto e com base na revisão da literatura especializada, pode-se observar que nos dois países se registraram etapas mais ou menos claras na relação entre obrigatoriedade escolar, universalização da educação básica, situação de pobreza do alunado e institucionalização de serviço/trabalho social no sistema educacional: etapa higienista, desenvolvimentista, autoritária e democratizante/neoliberal.

Palavras-chave: Direito à educação. Equipes multiprofissionais-multidisciplinares. Intersetorialidade. Legislação educacional. Processos de trabalho escolar-educacional.

1 "En los países en que el sentido de la educación estuvo articulado principalmente a una política de integración [nacional], el Estado cumplió un papel central en la creación de un sistema educacional más democrático, por medio de políticas de homogeneización e universalización de la educación (...). A diferencia de otros países, en Brasil el Estado nacional cumplió un papel subsidiario, quedando la educación asociada a la formación de élites e a las necesidades de un desarrollo industrial tardío e concentrado solamente en algunas regiones". (KRAWCZYK; VIEIRA, 2012, p. 150; traducción nuestra). 


\begin{abstract}
The demands for social work/service carried out by educational institutions in Argentina and Brazil are associated with the historical process of establishing compulsory schooling and the universalization of the right to education. This obligation was legislated for the first time in 1884 in Argentina by the Law number 1,420 and in the Federal Constitution of 1934 in Brazil, where it faced greater difficulties in order to be institutionalized due to the historic decentralizing force. In spite of this and based on a review of the specialized literature, we can observe that, in both countries, there were, more or less, clear steps in the relationship between compulsory schooling, universalization of basic education, student body poverty situation and institutionalization of social work/service in the educational system: hygienist, developmental, authoritarian and democratizing/neoliberal stage.

Keywords: Right to education. Multiprofessional-multidisciplinary teams. Intersectoriality. Educational legislation. Processes of school-educational work.
\end{abstract}

\title{
Antecedentes de la investigación
}

Creemos que los proyectos de investigación surgen fundamentalmente a partir de aquello que nos causa sorpresa. Nuestro actual proyecto de investigación no fue diferente ya que se influenció indirectamente por una investigación anterior (YANNOULAS, 2013a), que trataba la relación entre política educativa y pobreza. El desconocimiento mutuo de las profesionales de la educación y del trabajo social en Brasil se hizo evidente en el transcurso de aquella investigación, así como la necesidad de la gradual aproximación entre ambas.

En aquel momento (2012), organizamos un grupo de reflexión, entre asistentes sociales y maestras de escuelas, con la propuesta general de pensar conjuntamente sobre: 1) cómo la situación de pobreza de los alumnos de las escuelas públicas podría ser visibilizada, explicitada y trabajada en el contexto de las escuelas de educación básica y, paralelamente, 2) promover la aproximación de las áreas disciplinares Educación y Trabajo Social, en vista de la existencia del Proyecto de Ley n ${ }^{\circ} 3.688 / 2000^{2}$. Ambas áreas disciplinares (y otras) están involucradas con la garantía del Derecho a la Educación. La concretización de

2 El Proyecto de Ley n ${ }^{\circ} 3.688 / 2000$ establece la prestación de servicios de Psicología y de Servicio Social en escuelas públicas de educación básica. Otro proyecto importante sobre el asunto es la Propuesta de Enmienda a la Constitución n ${ }^{\circ} 13 / 2007$, que propone la garantía de los 
ese derecho demanda tanto la reglamentación y la instrumentalidad jurídica para comprobar la acción concreta a la que fue determinada por la legislación, como al conocimiento y la vivencia de la realidad cotidiana de los actores educacionales (CURY; FERREIRA, 2009). De manera recurrente, el grupo de reflexión también posibilitó algunos debates sobre la función de la escuela pública, su estructura y su funcionamiento, y sobre la intersectorialidad de las políticas públicas en el contexto escolar.

Las sesiones del grupo se constituían en momentos de discusión, relevamiento de problemas y construcción de estrategias para reducir la precarización de la enseñanza pública universal. En ese esfuerzo de sistematización, se destacó la centralidad del papel del Estado en el enfrentamiento de la cuestión social y la necesidad de fortalecimiento de la política social, con carácter universal y gratuito. La finalidad consiste en que la población en situación de pobreza tenga garantizado no solamente el acceso al Derecho a la Educación, sino también a las condiciones de permanencia y disfrute de ese derecho y actuación participativa en su consecución.

En el transcurso, percibimos sorprendidas que ciertas figuras profesionales, cotidianas en los equipos educacionales argentinos, no eran tan frecuentes en los brasileños. Las futuras trabajadoras sociales brasileñas, a pesar de que tal profesión fue inserida en el sistema educativo brasileño hace casi un siglo, tenían pocas oportunidades de apropiarse del debate de política educacional. Así, resolvimos abordar de manera más sistemática la relación entre Trabajo/ Servicio Social y Educación en el modelo educativo argentino y en el modelo educativo brasileño. La comparación con lo que ocurre en la Argentina nos pareció relevante y pertinente, en la medida en que pensábamos que los campos de prácticas y proyectos de formación en trabajo social incluyen la política educacional de manera estructurada. Esto se debe a los procesos históricos anteriores de establecimiento de la obligatoriedad escolar en la Argentina, el centralismo de las políticas educacionales en el surgimiento del sistema educativo nacional, la temprana universalización de la escuela primaria y la institucionalidad de los servicios sociales en el sistema educativo ${ }^{3}$.

alumnos de enseñanza fundamental y media de atendimiento por equipo formada por trabajadoras sociales y psicólogas (CFESS, 2015). Una visión crítica puede ser consultada en Almeida (2004).

3 Inicialmente, creíamos que la participación de las trabajadoras sociales en la vida educacional argentina estaba consolidada. A partir de la realización del proyecto actual, percibimos que, aun siendo mayor que en Brasil, esa participación todavía es materia de debate y construcción. Así, actualmente, proponemos diferenciar momentos históricos, niveles educativos, esferas geográficas específicas y formas de contratación al analizar la cuestión: la relación entre trabajo social y trabajo escolar sería desigual y combinada, compleja y contradictoria en ambos países. 
La investigación que desarrollamos actualmente tiene por objeto comprender y discutir el trabajo de los equipos multiprofesionales-multidisciplinares en ámbitos escolares y en la política educacional latinoamericana actual. Se localiza en la interface entre la Política/Gestión Educacional y la Política/Trabajo Social. Esta interface es de importancia crucial en el momento presente de la educación pública brasileña, pues:

a) Conforme a lo explicado anteriormente, está en debate en el Congreso Nacional de Brasil la ampliación de los equipos educativos con la potencial incorporación de profesionales de Psicología y Trabajo Social

b) La mitad de la población escolar brasileña puede ser considerada en situación de pobreza, siendo beneficiaria de algún programa de asistencia social, especialmente del Programa de Transferencia Condicionada de Ingresos-Bolsa Familia (DUARTE, 2012, 2013);

c) En la década de 1990, con la implementación del modelo educativo neoliberal y bajo la influencia de los organismos internacionales, el Estado brasileño amplió la cobertura de la educación básica (Ley de Directrices y Bases de la Educación Nacional $n^{\circ}$ 9.394/1996, Emenda Constitucional $n^{0} 59 / 2009$ y Ley $n^{\circ} 12.796 / 2013$ ), aunque con baja calidad, proceso que fue acompañado por el desarrollo de una serie de programas "voluntarios" en las escuelas, que tienen la intención de "resolver carencias" (SOUZA, 2013), es decir, derechos sociales establecidos en la legislación, que no son atendidos.

Diversos estudios señalan las dificultades para comprender de manera cabal la relación entre la política educativa y la situación de pobreza, por ser una relación intrínsecamente multideterminada (YANNOULAS, 2013a). Esos estudios nos llevaron a considerar necesaria la renovación del formato escolar (FRIGERIO, 2007, 2009), para alcanzar la universalidad de la política, más allá de la garantía de acceso o permanencia, con un trabajo multidisciplinar, multiprofesional e intersectorial que busque la calidad educativa principalmente. A partir del proceso paulatino de universalización de la educación básica, disputada por diversos movimientos sociales y entendida como elemento fundamental de la sociedad democrática, la parte más pobre de la sociedad entró y entrará masivamente en la educación pública de los diferentes niveles educativos, y

4 En este texto se optó explícitamente por utilizar el género gramatical femenino para hacer referencia a las categorías profesionales predominantemente ejercidas por mujeres, tales como maestra, pedagoga, profesora, psicóloga, trabajadora social, entre otras mencionadas (sobre el tema de la feminización de las profesiones y ocupaciones, ver Yannoulas (2013b)). Otra observación importante es que, en Argentina, la categoría profesional se denomina trabajadora social (y el curso de formación superior específico en trabajo social), y en Brasil asistente social (siendo el curso de formación superior específico en servicio social). 
nuevos conflictos y contradicciones se presentarán o agravarán el sistema. Esa situación es particularmente significativa, explicitando de manera contundente cómo el sistema educativo está atravesado por el modo de producción capitalista, entremezclando condiciones socioeconómicas y desempeño escolar a través de las condicionalidades educativas típicas de los programas focalizados contemporáneos (sobre ese tema ver SOARES, 2011).

En ese sentido, comprendemos que los equipos multiprofesionales-multidisciplinares serían en ese contexto una necesidad del trabajo escolar, pero también que el debate sobre la universalización de la educación básica debe incluir la problematización sobre el papel que los sistemas educativos asumen en la concretización de las políticas sociales focalizadas, a través de programas oriundos de la política de asistencia social. Uno de los desafíos que enfrentamos es la relación conflictiva entre la política educacional y la política de asistencia social en el contexto más amplio de la protección social. Pensar la materialización en la sociedad capitalista de la política educacional y la política de protección social (que incluye, pero no se limita a la asistencia social) implica considerar la existencia de conocimientos construidos socialmente, aunque distribuidos y apropiados de manera desigual, lo que reproduce privilegios y discriminaciones de diversa naturaleza 5 .

Así, una de las actividades centrales del proyecto de investigación, "El trabajo de los equipos multidisciplinares en las escuelas y en la Política Educacional", es recolectar información sobre equipos multidisciplinares en espacios escolares y en la política educacional, en el Brasil y en la Argentina, focalizando el análisis sobre el funcionamiento, los desafíos y las limitaciones en la implementación de políticas intersectoriales y trabajo multiprofesional en educación básica obligatoria (como derecho del ciudadano y deber del Estado) ${ }^{6}$.

5 Comprendemos la educación como derecho social, como dimensión de la vida social que resulta de luchas pasadas y presentes por el acceso a los conocimientos y a la legitimación profesional, derivados de la posesión de credenciales educacionales en una sociedad capitalista estructurada en clases sociales en conflicto y contradicción. En este sentido, consideramos que el sistema educativo está tensionado por la lógica capitalista de reproducción de privilegios, y que el derecho a la educación está permeado por intereses distintos y contradictorios.

6 En Argentina, la Constitución Nacional de 1994 consagra el derecho de enseñar y aprender en su artículo 14. La Ley de Educación Nacional n ${ }^{\circ} 26.206$ regula el mencionado derecho y establece la obligatoriedad escolar en la educación inicial (cinco años), la educación primaria (de los seis a los doce años) y la educación secundaria (de los trece a los diecisiete años). En el marco del Consejo Federal de Educación, fue aprobado el Plan Nacional de Educación Obligatoria y Formación Docente (Resolución CFE $n^{\circ}$ 188/12) para el quinquenio 2012-2016, instaurando objetivos, líneas de acción y logros esperados para la educación obligatoria, en términos de las responsabilidades nacionales, las responsabilidades compartidas entre nación y provincias y las responsabilidades provinciales. En Brasil, la educación básica obligatoria está regida por la Constitución Federal de 1988, la Enmienda Constitucional no 59 de 2009, por la Ley Federal no 9.394 de 1996 (ley de bases 
El proyecto de investigación adopta, como método, el estudio comparado, con una visión crítica y otorgándole una relevancia central. El proyecto se inició en 2014 y tiene previsto concluir en 2018. Actualmente cuenta con 15 participantes brasileños y argentinos, del área educacional y del trabajo social, que realizan actividades investigativas organizadas en diferentes componentes para abordar la complejidad de la problemática.

\section{Regulación de las políticas educativas: una visión comparada}

Desde el punto de vista metodológico, nuestra inspiración para el estudio crítico comparado actual surge de Krawczyk y Vieira (2012). Su originalidad reside en el análisis comparado y en la mirada multidisciplinar sobre las reformas educacionales implementadas en nuestra Región en la década comprendida entre 1995 y $2005^{7}$. De acuerdo con ellos, es posible observar la presencia de la matriz posburocrática de coordinación/regulación de las políticas educacionales, en las cuales el control jerárquico es sustituido por el autocontrol, la obligación de medios por la obligación de resultados, la regulación por la evaluación. La reforma global es sustituida por micro-mudanzas inducidas desde el centro hacia la periferia del sistema educacional, que poseen tres elementos destacados: la descentralización de la gestión del sistema educacional, la incorporación de la gestión privada en la gestión pública, la autonomía institucional como capacidad de administrar los problemas y captar recursos, combinada con la (co) responsabilización de los individuos y la movilización de la comunidad para la provisión y la calidad de la educación escolar. Es en ese contexto, los equipos multiprofesionales-multidisciplinares desarrollan sus actividades en ambientes escolares, con los límites que le son impuestos.

Las autoras abordan las características comunes de los países estudiados en lo que se refiere al proceso de regulación política y a la estrategia de cambio, a las transformaciones estructurales en la organización y en la gestión de los

y directrices de la educación nacional) y por el Plan Nacional de Educación (el último fue aprobado en 2014 y tiene vigencia hasta 2024), incluyendo la educación infantil (cuatro y cinco años), la fundamental (de los seis a los 14 años) y la media (de los 15 a los 17 años).

7 Entendemos la multidisciplinariedad como conexiones entre subcampos específicos de la ciencia, originados en disciplinas diferentes. Esta aproximación de subcampos disciplinares responde a una tentativa de llenar espacios vacíos, o sea problemas en que las disciplina por si solas no pueden resolver o explicar adecuadamente (DOGAN, PAHRÉ; 1993). Sobre la relación entre multidisciplina e intersectorialidad, ver Almeida (2014), Pereira (2014) y también Yannoulas, Souza (2016). 
sistemas educativos (organicidad del sistema educativo) y al papel de la evaluación en la reconfiguración de la relación Sociedad, Estado y Educación. Esa transformación está vinculada al nuevo modelo de integración social vigente, que desmonta el proyecto solidario propio del Estado de Bienestar y de desarrollo keynesiano, y rearticula los tres elementos bajo la perspectiva de la mundialización y de la competición en la nueva fase de acumulación capitalista (que acentúa las desigualdades). En el caso de América Latina, ese proceso del final del siglo XX debe ser especificado necesariamente. Los Estados de Bienestar no habían alcanzado el mismo grado de expansión y consolidación, comparados con los europeos, y gran parte de los países estaban pasando por un proceso de democratización después de dictaduras militares.

Sistematizando las semejanzas en las reformas educacionales latinoamericanas sobre la base de Krawczyk y Vieira (2012), A. Duarte (2010) propone la existencia de tres generaciones de reformas:

- La primera focalizó su atención en la ampliación del acceso/cobertura (en el caso de Brasil, la universalización de la enseñanza primaria, a diferencia de los otros países analizados que se concentraron en la expansión del nivel medio), y paralelamente en la descentralización (transferencia de responsabilidades y recursos);

- la segunda reforzó modos de control y responsabilización de las escuelas y de sus profesoras a través de los resultados educacionales (creación de sistemas centralizados de evaluación, divulgación de los resultados y desempeños educacionales; establecimiento de parámetros curriculares de aplicación obligatoria en territorio nacional, que estandarizan las prácticas docentes); y

- la tercera buscó redefinir las relaciones entre las responsabilidades públicas y el derecho a la educación, enfatizando las cuestiones relativas a la participación y control democrático de las políticas educativas (con dos consecuencias importantes: creación de instancias colegiadas $\mathrm{y}$ fortalecimiento de la figura del director o directora como gestora y no como profesora).

Si bien las recomendaciones de los organismos internacionales para nuestros países eran (y son) relativamente homogéneas, llevando a una reconfiguración de la relación entre Educación, Estado y Sociedad a través de la descentralización de la gestión educacional y la incorporación de la lógica empresarial en las políticas educativas, cada país procesó esas recomendaciones de manera específica, dependiendo del grado de desarrollo económico y tecnológico, de la concentración de la renta y de la universalización de los diferentes niveles educativos anteriormente alcanzados. Por ejemplo, los intentos en Argentina y Brasil, en lo referente a la redemocratización y recuperación del modelo de- 
sarrollista anterior a las dictaduras, fueron limitados por la privatización de la esfera pública y la interferencia de los organismos internacionales. Sin embargo, la descentralización de la gestión del sistema educativo (una de las directrices de la reforma posburocrática relacionada con la crisis fiscal de los gobiernos nacionales) tuvo un impacto diferente en la distribución de responsabilidades y dinámicas de negociación. Si en Argentina la descentralización ocurrió con una impronta provincial, en Brasil fue la municipalización el rasgo predominante.

\section{Contextualización histórica de la relación entre trabajo social y educación}

A partir de la investigación bibliográfica efectuada, comprendemos que tanto en Argentina como en Brasil el surgimiento de demandas al servicio/ trabajo social por parte de las políticas educativas y de las instituciones educacionales estuvo históricamente articulada a los procesos de implementación de la universalización del derecho a la educación y el establecimiento de la obligatoriedad escolar. Sin embargo, existen peculiaridades en cada caso, ya que en el Brasil la fuerza descentralizadora no habría permitido la difusión homogénea del servicio/trabajo social en las escuelas o en el sistema educativo. A pesar de ello, es posible visualizar que en ambos países hubo una secuencia de etapas más o menos definidas en la relación entre la educación obligatoria y la institucionalización de servicios/trabajos sociales en el sistema educativo: etapa higienista, desarrollista, autoritaria y democratizadora/neoliberal. ${ }^{8}$

Para comprender mejor estos supuestos preliminares, remontemos brevemente el proceso histórico de esa relación, presentando cada una de las etapas mencionadas. Según Dentz y Silva (2015), encontramos importante el modelo educativo norteamericano como antecedente histórico para entender la etapa higienista, que en 1906 estableció la figura de las "visitadoras" en los centros sociales, con el objetivo de construir un vínculo entre las escuelas y los barrios,

8 Siempre pensando que los procesos son complejos, que en cada etapa se verifica la persistencia de características de la etapa anterior, que los procesos constituyen una suerte de espirales dialécticas. Así, no está demás recordar que esa comparación supone una generalización al interior de países grandes y complejos, y que evidentemente hay excepciones o relativizaciones que pueden ser apuntadas para diferentes contextos territoriales. Si nuestra hipótesis inicial de investigación suponía una fuerte relación entre servicio/trabajo social y educación en la Argentina, al paso que pensábamos una incipiente relación en el Brasil, la investigación bibliográfica y el trabajo de campo realizado en ambos países nos mostró que ni todo está resuelto o consolidado en todo el territorio argentino, y ni todo es incipiente en algunos estados brasileños. 
constatar los motivos de la evasión escolar, el bajo desempeño escolar y la falta de "adaptación" a la escuela del cuerpo discente.

La relación entre Trabajo Social y Educación en Argentina y en Brasil se remonta a la década de $1930^{\circ}$ y se desarrolla especialmente a partir de 1940, con las "visitadoras sociales", en el contexto de una perspectiva higienista, moralizadora de las clases sociales subalternas. El higienismo acompañó las corrientes inmigratorias y se proponía controlar y evitar las enfermedades en los centros urbanos en crecimiento, a partir de una educación sanitaria de carácter conservador. Según Witiuk:

Siendo la escuela uno de los aparatos privados significativos para el mantenimiento de la hegemonía, el Trabajo Social será requerido para el ejercicio de las atribuciones que armonizan las relaciones en el proceso de vigilancia de la moral y de la sociabilidad de las familias empobrecidas. Las modalidades de intervención serán objetivadas para la integración social de los individuos en el espacio escolar, comunitario y doméstico, reforzando la identidad subalterna por medio de la inculcación de valores dominantes y de la interferencia en su modo de vida con la adopción de conductas de comportamiento adecuado a los parámetros morales. (WITIUK, 2004, p. 37, traducción nuestra).

Ese modelo de servicio social escolar brasileño fue inspirado a partir del modelo anteriormente mencionado de Estados Unidos, basado en la intervención en casos individuales, y con la pretensión de detectar las causas familiares para los problemas escolares de los alumnos de familias en situación de pobreza, elaborando informes sociales y promoviendo el ajuste o adaptación al modelo escolar hegemónico. La literatura señala dos experiencias de servicio social educacional en la década de 1940, en los estados de Rio Grande do Sul y Pernambuco, en las cuales la función era resolver problemas como evasión, repitencia, ausentismo "y demás malestares escolares", "ajustando a los desajustados y ayudando a los necesitados" (DENTZ; SILVA, 2015, traducción nuestra).

Esa relación se verifica también en Argentina con la creación del "Cuerpo de visitadoras” de Higiene Escolar por el Consejo Nacional de Educación

9 En Argentina, en 1924 se creó la primera Carrera de visitadoras de higiene social, y, en 1930, la Escuela de Servicio Social, ambas dependientes de la Universidad de Buenos Aires. En Brasil, la primera escuela fue creada en São Paulo, vinculada al Centro de Estudios de Acción Social, que más tarde se transformó en la actual Facultad de Servicio Social de la Pontifícia Universidade Católica (PUC) de São Paulo. 
(ACOSTA et al., 2008). En la década de 1940, algunas provincias argentinas crearon áreas específicas de Psicología y Asistencia Social Escolar en la estructura del sistema educativo provincial (siendo pionera la Provincia de Buenos Aires). (CORROSA; LÓPEZ; MONTICELLI, 2006).

Oliva (2006) afirma que la función educativa de los profesionales del trabajo/servicio social estuvo desde su origen marcada por profundas contradicciones, ya que las cuestiones de educación sanitaria eran preocupaciones de la burguesía (trasladando la verdadera necesidad de inversión estatal para mejorar las condiciones de vida de los asalariados para cada hogar), y también reivindicaciones de los trabajadores (enfoque socialista reformista ligado a la elevación de las condiciones de existencia de la clase trabajadora):

El espacio ocupacional del trabajo social, desde su origen, se fue estableciendo en torno a las funciones de asistencia, gestión y educación, presentándose simultáneamente y con distintos grados de articulación. Estas funciones se han orientado con objetivos de modificación sobre las prácticas y conductas de las clases trabajadoras; por ello se desarrollan de diversas formas e incluso con direccionalidades opuestas. (OLIVA, 2006, p. 75, grifos de la autora).

La influencia del modelo desarrollista llevó, en ambos países, a la instauración de la reflexión y la intervención en torno a la relación educación-trabajo, con la creación de los servicios de orientación profesional. La perspectiva que predominaba en ese momento histórico era la de la teoría del capital humano, reservando el trabajo/servicio social en la educación como un tipo de intervención residual, tecnicista, con miras a la alfabetización y orientación profesional de los futuros trabajadores, y con foco en la gestión de eficiencia y racionalización de costos y acciones. Especialmente tuvo influencias la realización de eventos promovidos por organismos internacionales (ONU, OEA), como el III Congreso Panamericano de Servicio Social realizado en Puerto Rico en 1957. Uno de sus resultados fue la homogeneización de la terminología del área, incluyendo entre los tópicos el Servicio Social Escolar:

Servicio Social escolar, school social work. Es lo que se presta en los establecimientos de enseñanza y que pretende atender problemas o circunstancias que perturban o impiden la utilización de las potencialidades de los educandos. (III ${ }^{\circ}$ Congreso Pan-Americano de Servicio Social, 1957, p 132; traducción nuestra de texto extraído de Witiuk, 2004). 
Argentina fue uno de los primeros países en incluir la entonces denominada "Orientación Profesional" entre los derechos de la Educación y la Cultura, con el artículo 37 de la Constitución Nacional promulgada en 1949 durante el gobierno peronista, y se crea la Dirección de Psicología Educativa y Orientación Profesional - la cual en 1953 incorporará el área de asistencia social. Según Acosta et al. (2008) la reestructuración realizada provocó que se intensificara la coordinación con otros servicios asistenciales, la utilización de la encuesta social, siendo "lo social" comprendido todavía como un problema individual y la tarea de la trabajadora social la de medir y diagnosticar. En la segunda mitad del siglo, las instituciones que alentaron los desarrollos de la Orientación se multiplicaron, con la creación de carreras de Psicología en varias universidades estatales y privadas, y también en los profesorados de Psicología y Psicopedagogía. Durante estos años, las teorías de los rasgos y factores, la difusión y aplicación de la psicometría y el modelo fordista-taylorista de producción influyeron en las prácticas orientadoras, bien como el psicoanálisis. En 1965, la Universidad de Buenos Aires, a través de su Departamento de Orientación Vocacional, organiza las Primeras Jornadas de Orientación Vocacional ${ }^{10}$.

Para el caso brasileño, en 1961 fue aprobada la Ley n ${ }^{\circ} 4.024$ de Directrices y Bases de la Educación, que estableció la asistencia social escolar, dando origen a diversos proyectos de ley estaduales para su implementación. La ley aprobada en ese momento no contenía algunos de los elementos fundamentales defendidos por los movimientos en defensa de la educación pública, durante los 13 años que duró el debate político-ideológico del proyecto de ley. En la reglamentación de la asistencia social escolar en la esfera estadual (provincial), pueden observarse diferentes perspectivas contradictorias sobre cómo implementar la asistencia social escolar prevista en la ley federal. Por ejemplo, en Santa Catarina, la ley estadual preveía actuación en situaciones consideradas de "desvío, defecto o desajuste”; en otros casos, como Paraná, se establecieron competencias más amplias y complejas, con trabajo colaborativo con otros profesionales de la educación y con la comunidad. Paralelamente, se desarrollan proyectos de carácter experimental de las municipalidades con apoyo de organismos internacionales (como la Fundación Ford en la Municipalidad de Guanabara/Rio de Janeiro), donde queda clara la perspectiva de defensa de los intereses del capital a través de acciones que garanticen la alfabetización y la formación profesional de los futuros trabajadores (WITIUK, 2004). También es importante mencionar el peculiar desarrollo y consolidación de capacitación profesional en el Brasil, fuera

10 Para más detalles sobre la cuestión de la orientación vocacional y profesional en la Provincia de Entre Ríos, ver Costa y Lenarduzzi, 2005. 
del sistema educativo y a través del denominado "Sistema S" 11 (CUNHA, 2000; GIROLAMI, 2014). Este sistema incorporó diferentes profesionales para actuar en la relación formación-trabajo, entre ellos las asistentes sociales.

La década de 1970, a través de la denominada reconceptualización y con fuerte influencia de las ideas de Paulo Freire, fue crucial para el trabajo/ servicio social en la Región, trayendo serios cuestionamientos al ejercicio profesional tradicional y, especialmente, al proyecto ético-político. Surgió, entonces, un nuevo enfoque de intervención profesional de ruptura, cambiando al profesional paternalista superior por una relación de igualdad y diálogo, y con una consecuente crítica a los métodos clásicos de trabajo social de caso, con la consolidación de trabajos de grupos y de comunidad con intención de defender el punto de vista de la clase trabajadora. Simultáneamente, se registró una preocupación por la definición del papel de los trabajadores sociales en la educación y en los equipos de orientación educacional, con un aumento de la cantidad y extensión de los servicios sociales ofrecidos (asistencia directa al escolar y promoción comunitaria, tendientes a erradicar el analfabetismo y evitar la repitencia), pero en un contexto político cada vez más desfavorable por la instauración de procesos políticos autoritarios y represivos en ambos países.

Esto generó tensiones específicas, porque si la tendencia de la categoría profesional indicaba abertura, trabajo comunitario, salida de la atención individual, entre otros aspectos, el contexto político limitaba de manera extraordinaria la posibilidad de que esto efectivamente ocurriese en las escuelas. Las dictaduras militares impondrán retrocesos de diversa naturaleza, con desmantelamiento de la estructura institucional, pérdida de personal, persecución política, atomización del trabajo en equipo, y foco en el fracaso escolar y el trabajo de los profesionales no-docentes en la readaptación de los "alumnos problema" con especificaciones muy claras sobre las competencias y atribuciones del trabajo social: cuidar del ausentismo, el abandono y la repitencia escolares. Hubo, naturalmente, espacios de resistencia y conflicto, pero no pudieron prosperar en un clima tan hostil.

La reflexión crítica sobre la relación entre políticas educacionales y políticas sociales verá y vivirá nuevos horizontes a partir de los procesos de democratización vivenciados en ambos países a partir de la segunda mitad de la década de 1980. También fueron aprobadas convenciones internacionales de derechos de la infancia y la adolescencia (Convención de los Derechos del Niño aprobada en la Asamblea de las Naciones Unidas de noviembre de 1989),

11 "Sistema S" denomina el conjunto de instituciones de categorías profesionales: Servicio Nacional de Aprendizaje Comercial, Industrial, Rural, del Transporte, del Cooperativismo, entre otros. Los recursos, establecidos en porcentaje sobre los salarios pagos por las empresas, deben ser aplicados para financiar actividades de capacitación profesional, salud y ocio. 
posteriormente refrendadas en los dos países (en Argentina Ley n 23.849 y en Brasil Ley ${ }^{\circ}$ 8069), estableciendo un marco importante para la protección integral de los niños y adolescentes de ambos sexos.

Infelizmente, el movimiento político democratizador (que buscaba recuperar la función social y política de la escuela) fue paralelo a la implementación de un modelo económico neoliberal, que limitó las posibilidades de expansión de esa relación entre políticas educacionales y políticas sociales. Los nuevos modos de regulación posburocrática de los sistemas educativos impusieron serias limitaciones a la función preventiva y propositiva/promotora del trabajo social en la educación, conformándose un abordaje salvacionista de la actuación profesional y limitado a la relación entre pobreza y educación (LESSA, 2013), que reduce las competencias del trabajo social al sacar su foco de la cuestión social en sus expresiones escolares (SANTOS, 2015), y por veces le otorga una impronta "policialesca-controladora" al servicio de la "eficiencia" de los programas sociales focalizados.

La nueva modalidad de regulación y el nuevo contexto económico impusieron una función paliativa a los equipos de orientación, principalmente reduciendo la actuación profesional cada vez más a la administración y gestión de los programas de transferencia de ingresos con condicionalidades educacionales y a la articulación con otras políticas sociales y otros profesionales requeridos para la educación inclusiva de alumnado con necesidades especiales. De alguna manera podemos afirmar que, si se registra falta de claridad sobre la tarea de los equipos, hay también una demanda creciente hacia los equipos para que resuelvan o actúen sobre las consecuencias de la implementación de modelos socioeconómicos excluyentes y las expresiones de la cuestión social en el cotidiano educativo.

La inserción en el cuadro del personal del sistema educativo de las trabajadoras sociales y otras profesionales (como psicólogas y psicopedagogas) se da, principalmente, en función de la obligatoriedad de la educación básica. Las tareas de los equipos de apoyo y orientación se establecen en Argentina en el marco de la Ley de Educación Nacional, los Acuerdos Federales (Resoluciones Consejo Federal de Educación n ${ }^{\text {os }}$.93/09, 174/12, 188/12, 217/14), las Leyes de Educación Provinciales y las normas que encuadran la función sustantiva de estos equipos ${ }^{12}$. Las figuras institucionales denominadas para el caso entrerriano son equipos de orientación educativa (EOE), para el nivel secundario; y servicio

12 Para más detalles sobre la cuestión, ver ARGENTINA. Consejo Federal de Educación. Resolución $n^{\circ} 239$ de 2014 - Establece Pautas y Criterios para los Equipos de Apoyo y Orientación Escolar; y también ARGENTINA. Ministerio de Educación de la Nación, Los Equipos de Orientación en el Sistema Educativo - la dimensión institucional de la intervención, 2014. 
de apoyo interdisciplinario educativo (SAIE), para el nivel inicial, especial y primario. Entretanto, es necesario analizar cómo se ponen en juego las normativas nacionales y provinciales en los contextos institucionales específicos.

Su sede física se establece en las direcciones departamentales, aun cuando eventualmente esos equipos multiprofesionales-multidisciplinares pueden funcionar dentro de una institución escolar. Por ley, los equipos atienden las demandas de un grupo de escuelas, pero su capacidad de intervención institucional, a juicio de los participantes del taller realizado en la UNER bien como de las fuentes y bibliografía consultadas, estaría muy asociada al estilo de conducción de cada escuela y/o dirección educativa y restringida por un enfoque reparatorio predominante en las políticas sociales, actuando sobre los daños provocados por el propio sistema de acumulación que maneja la exclusión social como parte de su lógica intrínseca (CLEMENTE, 2014).

Actualmente los equipos están abocados prioritariamente a las trayectorias escolares, focalizando en el fortalecimiento de las trayectorias escolares diversificadas a través de la generación de mejores condiciones institucionales para la enseñanza y el aprendizaje. Desde el abordaje de lo colectivo-institucional, también se dedican a la promoción de la convivencia y el abordaje de la conflictividad social en las instituciones educativas, pero constatamos en las expresiones de las trabajadoras sociales que participaron de los eventos la existencia de resistencias de la institución escolar con relación a la implementación de los acuerdos de convivencia, inclusive el hecho que los directivos no demandan esa tarea a los equipos y/o que los diferentes profesionales que componen los equipos no logran consensuar internamente las propuestas por partir de formaciones y ejercicios profesionales muy diferentes.

Con relación a las tareas específicas de los equipos, se detalla por norma que:

[...] implica diseñar, implementar y acompañar -junto con supervisores, directivos y docentes- procesos favorables a la enseñanza y aprendizaje, para todas/os las/os alumnas/os, lo que también incluye atender situaciones particulares de estudiantes y propias de los grupos de estudiantes, así como colaborar en proyectos y propuestas de enseñanza, de convivencia, tutoriales, etc., generadoras de mejores condiciones institucionales. Las tareas de diagnóstico y de clínica, ante situaciones individuales de los estudiantes, no pueden ser llevados a cabo por los docentes, directivos o profesionales que se encuentran desempeñando su rol en el ámbito escolar. El campo disciplinar de procedencia de los integrantes de los Equipos de Apoyo y Orientación dará elementos para orientar a los docentes sobre las prácticas de enseñanza y a los padres sobre los particulares procesos 
de aprendizaje de sus hijos. (ARGENTINA. Ministerio de Educación de la Nación, 2014, p. 3).

La misma norma establece que los equipos, para cumplir sus tareas y de acuerdo con la realidad local, pueden reunir a profesionales de diversas formaciones de origen (psicólogas, psicopedagogas, trabajadoras sociales, antropólogas, sociólogas, pedagogas, fonoaudiólogas, médicas con orientación comunitaria, entre otras profesiones).

Un relevamiento exploratorio para el caso brasileño, realizado por Lins (2016), parecería indicar una fuerte asociación entre la incorporación de otras profesionales al equipo multidisciplinar-multiprofesional y la implementación de programas federales de carácter intersectorial, tales como el programa bolsa-familia o la política nacional de asistencia estudiantil (modelo de regulación posburocrático). Aunque se tenga registro de trabajadoras sociales actuando en la educación desde la década de 1930, es recién a partir de los años 1990 que se puede vislumbrar la inserción de esta profesional en el campo escolar de manera más consistente, en un contexto histórico-económico de desmonte de lo público (Estado), ampliación de la responsabilidad familiar y de las instituciones del tercer sector en la provisión de ese derecho.

En cuanto a la actuación del Trabajo Social en la Educación brasileña, no hay consenso dentro de la categoría sobre cuál sería el papel de esa profesional en los equipos escolares, constituyendo su definición uno de los grandes desafíos (CFESS, 2013). La actuación es heterogénea en cuanto a los niveles y las funciones. Por ejemplo, ellas ya están presentes en el trabajo educativo respecto al acceso y permanencia en el nivel superior y en la enseñanza técnica de los institutos federales, integrando los equipos que gestionan la asistencia estudiantil con base en la Política Nacional de Asistencia Estudiantil. En algunas unidades de la federación, ellas tienen inserción en las escuelas primarias o en las secretarías municipales o provinciales de educación o de asistencia social, actuando junto a la tríada, escuela/familia/instituciones, garantes de derecho (SOUZA, 2008; MARTINS, 2011, 2012; SILVA, 2012).

Según Yannoulas y Souza (2016), la reglamentación del sistema educativo brasileño (la Constitución Federal de 1988, la Ley de Directrices y Bases de la Educación Nacional n ${ }^{\circ}$ 9.394/1996, la Ley n ${ }^{\circ} 11.738 / 2008$, que creó el Piso Salarial Profesional Nacional para los profesionales del magisterio público de Educación Básica, y la Ley n n 12.014/2009, que estableció las categorías de trabajadoras que se deben considerar profesionales de la educación), se entiende por trabajadoras de la educación a todas las personas que actúan en la institución escolar. Sin embargo, como resultado de la definición por ley del piso salarial, 
fue necesario establecer cuáles serían las trabajadoras que efectivamente tenían el derecho de exigir tal piso. Así, son consideradas profesionales de la educación aquellas personas que desarrollan actividades de docencia o de soporte pedagógico a ella. En esta categoría se incluye expresamente:

[..] dirección o administración, planeamiento, inspección, supervisión, orientación y coordinación educacionales, ejercidas en el ámbito de las unidades escolares de educación básica, en sus diversas etapas y modalidades, con la formación mínima determinada por la legislación federal de directrices y bases de la educación nacional. (BRASIL, 2008, traducción nuestra).

Por último, las profesionales del magisterio son solamente las trabajadoras directamente involucradas en el ejercicio de las actividades de docencia. Sería necesario, por lo tanto, debatir en cuál categoría podrían ser incluidas las trabajadoras sociales y las psicólogas, en el caso de su actuación en las escuelas brasileñas; $y$, más específicamente, cuál sería el aporte esencial de esas profesionales en el ambiente escolar y en la educación básica, como también el proceso de trabajo a ser desarrollado por ellas como parte integrante del equipo escolar ${ }^{13}$.

Según Yannoulas y Souza (2016), en Brasil no existe una legislación unívoca en cuanto a la composición de los equipos escolares de la educación básica, siendo muy grande la variación entre los estados y los municipios. Cada red municipal o estadual organiza los equipos de acuerdo con su historia, tradiciones y tensiones. Hay estados, como, por ejemplo, Santa Catarina, que tiene supervisor, orientador y director. Otras poseen director, director auxiliar y coordinador pedagógico. El número de profesionales también varía de red en red. En general, se define el porte del equipo a partir del número de alumnos existente en la escuela o en la red. Pero esto es establecido en cada red por los respectivos Consejos Estaduales y Municipales de Educación. Además de la dirección y coordinación pedagógica en cada escuela, las secretarías de educación municipales y estaduales, en general, poseen un equipo que acompaña, auxilia y controla la red de escuelas de su jurisdicción. En municipios muy pequeños, muchas veces, no hay secretaría de educación, sólo un Departamento de Educación, dentro de alguna secretaría mayor. Esa falta de uniformidad administrativa

13 Un análisis de la categoría proceso de trabajo aplicada a los equipos escolares y más específicamente a la formación profesional e intervención de las trabajadoras sociales y las maestras/ pedagogas puede ser consultada en Yannoulas, Rocha y Soares (2016). 
y de gestión pedagógica al interior de las redes municipales y estaduales, que componen el sistema, constituye un serio obstáculo para el desarrollo de la anhelada educación básica de calidad.

\section{Consideraciones finales}

A modo de conclusión nos gustaría dejar planteadas cuestiones que deberíamos pensar en la intervención de los grupos multiprofesionales y multidisciplinares en las escuelas. Como expresamos con anterioridad a partir del proceso paulatino de universalización de la educación básica los sectores más vulnerables de la sociedad entraron masivamente en la educación pública, por lo tanto, nuevos conflictos y contradicciones se presentan o agravan en el sistema educativo. La problemática social irrumpe y se filtra permanentemente en las instituciones educativas: violencia social y familiar, trabajo infantil, fragilidad en los vínculos familiares, precariedad habitacional y laboral, embarazo adolescente, consumo problemático de sustancias, desidia, apatía, dificultades en la convivencia ingresan a las aulas de la mano de niños, adolescentes y sus familias, situaciones para las que los equipos multidisciplinares y específicamente las trabajadoras sociales están llamadas a intervenir. Coincidimos con Piana (2009), que expresa lo negativo que es para la trabajadora social la consolidación de su presencia asociada a prácticas paliativas y asistenciales en el universo escolar.

Innegablemente, los programas de transferencia condicionada de ingresos, como el Bolsa Familia (Brasil) o la Asignación Universal por Hijo (Argentina), están generando un fuerte impacto en el sistema escolar. Han ingresado a la escuela un conjunto de niños y adolescentes a quienes no se los estaba esperando y que jamás han concurrido a ella o bien que estaban fuera. Se desvelan, así, una serie de marcas originarias y desafíos presentes en el sistema educativo, ya sea de orden de funcionamiento (un sistema educativo fragmentado), de orden material (la necesidad de cubrir una demanda mayor tensiona la capacidad de los establecimientos educativos para responder, lo cual supone inversiones en infraestructura, recursos, etc.) y de orden pedagógico (los niños, niñas y adolescentes que se incorporan al sistema educativo son mayoritariamente de sectores que sufren distintas formas de exclusión y discriminación, también al interior de las escuelas) ( KAPLAN, 2010).

Por tanto, los programas de transferencia condicionada de ingresos constituyen tanto una oportunidad como un desafío para el sistema educativo. Se trata de una oportunidad en la medida en que ayuda al cumplimiento de leyes 
educativas y que sirve como un instrumento de gestión para reforzar y potenciar las políticas que se están llevando a cabo; y un desafío porque tensiona e interpela al sistema en su conjunto. Sin embargo, puede llegar a ser una tensión positiva, puesto que permitiría dar un salto cualitativo importante en la medida en que la escuela no desvirtúe su esencia que es educativa; además de que las trabajadoras sociales y otras profesionales escolares no restrinjan sus competencias.

En cuanto a los estudiantes marcados por procesos de exclusión de diversos tipos, estos tienden a percibirse a sí mismos como causa última de su propio fracaso; se desacreditan como producto del descrédito del que han sido objeto (KAPLAN, 1992; FILMUS et al., 2001; GIROLAMI, 2015). El ausentismo, la repitencia y la sobre edad afectan las trayectorias escolares de un importante porcentaje de niños y adolescentes en las instituciones educativas, acercándolos a la frontera del abandono escolar y la posterior deserción. Al mismo tiempo, se pone de relieve el valor insoslayable que posee la escuela para los niños y adolescentes atravesados por los condicionamientos materiales y simbólicos de la pobreza. Fuera de la exclusión social, en ciertas instituciones escolares aprenden a revalorizarse como sujetos de derecho, lo cual resignifica su propia valía social y escolar.

En este contexto resulta estratégica la intervención de los equipos multidisciplinares y multiprofesionales, donde el trabajo/ servicio social está inmerso. Uno de los principales ejes articuladores de esta dimensión en la intervención en escuelas es el carácter subsidiario que asume "lo social" a favor de "lo educativo". Es decir, "lo social" está pensado en pos de que la institución educativa desarrolle sin obstáculos la función social a la que es llamada. Se interviene sobre las "problemáticas sociales" que pudieran afectar el aprendizaje de los niños (ACOSTA et al., 2008).

Estas problemáticas que interfieren en el aprendizaje de los niños son macrosociales, y, por lo tanto, deberían ser abordadas de manera integral. Es por ello que la tarea efectivamente realizada por la trabajadora social en la escuela es solo una arista de la resolución de esas problemáticas. Esto constituye un límite objetivo de la intervención y de las posibilidades que se tienen para intervenir. De esta manera, la intervención es una respuesta determinada que se da ante los procesos sociales y las transformaciones sociales, pero estas respuestas no son siempre las mismas, son resultado de una realidad histórica particular, al tiempo que intervienen sobre esa realidad (ACOSTA et al., 2008; PEREIRA, 2010). No existe una única manera de intervenir, ya que la intervención es un complejo de variables que se articulan en un movimiento dinámico y permanente, configurando una opción para el profesional. Esta opción se va a determinar teniendo en cuenta el contexto, es decir, sus características y determinaciones históricas, sociales, culturales, económicas y políticas. 
La trabajadora social interviene en la formulación e implementación de proyectos que aborden estas situaciones desde la singularidad de estos sujetos con propuestas interdisciplinarias dentro de la institución escolar, así como también a partir del trabajo en red con instituciones barriales, donde la escuela se encuentra emplazada. En este sentido, resulta muy interesante el concepto de las políticas públicas entendidas como acuerdos entre el Estado y la Comunidad (CANDIANO, 2010). Ese lugar intermedio es lo que define a la política pública. Son los acuerdos y su materialización, que se alcanzan entre el Estado y la Sociedad, lo que hace que la política pública sea creíble, sustentable, clara y aceptada (CANDIANO, 2010).

Compartimos con Imbert (2012), que es necesario seguir apostando en la escuela. Y frente a la realidad de la escuela hoy, compartida por ambos países, la incorporación de los equipos multidisciplinares como política de Estado en Brasil es imperante, mientras que en la Argentina es necesario la profundización del rol de los mismos y la construcción de estrategias de intervención, que den cuentan de la compleja realidad que tienen que abordar.

\section{REFERENCIAS}

ACOSTA, M. L. et al. La Intervención Profesional en Escuelas: viejas demandas, viejas estrategias, nuevas situaciones. Buenos Aires: Universidad de Buenos Aires, Facultad de Ciencias Sociales, Trabajo Social, 2008.

ALMEIDA, N. L. T. Parecer sobre os projetos de lei que dispõem sobre a inserção do Serviço Social na Educação. Brasília: CFESS, 2004.

ALMEIDA, N. L. T. Descentralização e intersetorialidade: desafios para a consolidação da política pública de educação. In: MONNERAT, G. L.; ALMEIDA, N. L. T.; SOUZA, R. G. S. A intersetorialidade na agenda das politicas sociais. Campinas, SP: Papel Social, 2014. p. 231-260.

ARGENTINA. Consejo Federal de Educación. Resolución n 239 de 2014 - Establece Pautas y Criterios para los Equipos de Apoyo y Orientación Escolar. 2014.

ARGENTINA. Ministerio de Educación de la Nación. Los Equipos de Orientación en el Sistema Educativo - la dimensión institucional de la intervención, 2014.

CANDIANO, R. Asignación Universal por Hijo. Buenos Aires: Ciclo de conferencias organizado por la Asociación Argentina de Políticas Sociales AAPS, la Red Argentina de Ingreso Ciudadano REDAIC, 2010. 
CLEMENTE, A. (Coord.). Territorios urbanos y pobreza persistente. Buenos Aires: UbaSociales y Espacio Editorial, 2014.

CORROSA, N.; LÓPEZ, E.; MONTICELLI, J. M. El trabajo social en el área educativa. Desafíos y perspectivas. Buenos Aires: Espacio, 2006.

COSTA, Z. U.; LENARDUZZI, Z. V. et al. Orientación Vocacional y Sistema Educativo en la Provincia de Entre Ríos. Ciencia, Docencia y Tecnología, v. 30, p. 49-89, 2005.

CUNHA, L. A. O ensino industrial-manufatureiro no Brasil. Revista Brasileira de Educação, n. 14, p. 89-193, 2000.

CURY, C. R. J.; FERREIRA, L. A. M. A judicialização da educação. Revista CEJ, Brasília, Ano XIII, n. 45, p. 35, abr./jun. 2009.

DENTZ, M. von; SILVA, R. R. D. da. Dimensões históricas das relações entre educação e Serviço Social: elementos para uma revisão crítica. Serviço Social e Sociedade. São Paulo, n. 121, p. 7-31, jan./mar. 2015.

DOGAN, M.; PAHRÉ, R. Las Nuevas Ciencias Sociales. Mexico: Editorial Grijalbo, 1993.

DUARTE, A. Tendências das Reformas Educacionais na América Latina para a Educação Básica nas décadas de 1980 e 1990. In: FARIA FILHO, L. M. et al. Reformas Educacionais no Brasil: Democratização e qualidade da escola pública. Belo Horizonte: Maza Edições, 2010. p. 161-185.

DUARTE, N. de S. Política Social: um estudo sobre educação e pobreza. Tese (Doutorado em Política Social) - Programa de Pós-Graduação em Política Social, Universidade de Brasília, Brasília. 2012.

DUARTE, N. de S. O impacto da pobreza no Ideb: um estudo multinível. Revista Brasileira de Estudos Pedagógicos, v. 94, p. 343-363, 2013.

FILMUS, D. et al. Cada vez más necesaria, cada vez más insuficiente. Escuela media y mercado de trabajo en épocas de globalización. Buenos Aires, Santillana, 2001.

FRIGERIO, G. Inventarios. Argumentos para ampliar lo pensable. In: BAQUERO, R.; DICKER, G.; FRIGERIO, G. (Comps.). Las formas de lo escolar. Buenos Aires: Del Estante, 2007. p. 323-340.

FRIGERIO, G. Obstinaciones duraderas. In: ADESU. Educación social: acto político y ejercicio profesional. Montevideo: MEC/ADESU, 2009. p. 41-75.

GIROLAMI, M. C. Educação e trabalho: um olhar dos jovens de baixa renda do Brasil e da Argentina. Tese (Doutorado em Ciências Sociais) - Programa de Pós-Graduação em Ciências Sociais, Universidade de Brasília, Brasília. 2014.

GIROLAMI, M. Educación y Trabajo. Revista perspectiva de Políticas Públicas, Año 5, n. 9, p. 53-88, Jul./dic. 2015. 
IMBERT, L. Debemos Seguir Apostando a las Potencialidades de la Escuela. Entrevista. A lo Hecho Dicho. Facultad de Trabajo Social de la UNER, Paraná, n. 6, sept. 2012.

KAPLAN, C. V. Buenos y malos alumnos: descripciones que predicen. Buenos Aires, Aique, 1992.

KAPLAN, C. V. Asignación Universal por Hijo. Buenos Aires: Ciclo de conferencias organizado por la Asociación Argentina de Políticas Sociales AAPS, la Red Argentina de Ingreso Ciudadano REDAIC, 2010.

KRAWCZYK, N. R.; VIEIRA, V. L. Uma perspectiva histórico-sociológica da reforma educacional na América Latina: Argentina, Brasil, Chile e México nos anos 1990. Brasília/DF: Liberlivro, 2012.

LESSA, S. E. do C. A educação contemporânea, o combate à pobreza e as demandas para o trabalho do assistente social. Serviço Social e Sociedade, n. 113, p. 106-130, jan./mar. 2013.

LINS, H. H. F., Relatório final individual-Edital 2015 Proic/Cnpq/UnB - Legislações sobre as equipes multidisciplinares nas escolas e na política educacional: um estudo comparado. Brasília/DF: UnB/TEDis, agosto 2016 (mimeo).

MARTINS, E. B. C. Implicações da ética profissional do assistente social no espaço educacional. Textos \& Contextos (Porto Alegre), v. 10, n. 1, p. 44-55, jan./jul. 2011.

MARTINS, E. B. C. Educação e Serviço Social - Elo para a construção da cidadania. São Paulo: Editora da UNESP, 2012.

OLIVA, A. A. Antecedentes del Trabajo Social en Argentina: asistencia y educación sanitaria. Trabajo Social, Revista del Departamento de Trabajo Social, Facultad de Ciencias Humanas, Universidad Nacional de Colombia, n. 8, p. 73-86, 2006.

PEREIRA, P. A. P. Entrevista. Politizando - Boletim do Núcleo de Estudos e Pesquisas em Política Social. Brasília, Número sobre Educação e Política Social, 2010.

PEREIRA, P. A. P. A intersetorialidade das políticas sociais na perspectiva dialética. In: MONNERAT, G. L.; ALMEIDA, N. L. T.; SOUZA, R. G. S. A intersetorialidade na agenda das políticas sociais. Campinas, SP: Papel Social, 2014. p. 23-39.

PIANA, M. C. A construção do perfil do assistente social no cenário educacional. São Paulo: Editora da Unesp, 2009.

SANTOS, F. H. O exercício profissional de assistentes sociais em escolas públicas. Educação básica Revista, v. 1, n. 2, p. 61-74, 2015.

SILVA, M. M. J. da (Org.). Serviço Social na Educação: teoria e prática. Campinas/SP: Papel Social, 2012.

SOARES, K. J. Pobreza e educação formal: a relação entre pobreza e política educacional no Distrito Federal. Dissertação (Mestrado em Política Social) - Programa de Pós-Graduação em Política Social, Universidade de Brasília, Brasília. 2011. 
SOUZA, I. de L. Serviço Social na Educação - saberes e competências necessárias no fazer profissional. Tese (Doutorado em Educação) - Programa de Pós-Graduação em Educação, Universidade Federal do Rio Grande do Norte, Rio Grande do Norte. 2008.

WITIUK, I. L. A Trajetória Sociohistórica do Serviço Social no Espaço da Escola. Tese (Doutorado em Serviço Social) - Programa de Pós-Graduação em Serviço Social da Pontifícia Universidade Católica de São Paulo, Puc/SP, 2004.

YANNOULAS, S. C. (Coord.), Política Educacional e Pobreza. Múltiplas Abordagens para uma Relação Multideterminada. Brasília/DF: Liberlivro, 2013a.

YANNOULAS, S. C. (Coord.). Trabalhadoras - Análise da Feminização das Profissões e Ocupações. Brasilia: Abaré, 2013b.

YANNOULAS, S. C.; SOUZA, S. Equipes Escolares: Multidisciplina e Intersetorialidade. Brasília/DF: UnB/TEDis, abril/2016 (mimeo).

YANNOULAS, S. C.; ROCHA, A. P. de M. O.; SOARES, K. Processos de Trabalho na Escola e na Politica Educacional - Revisão de Literatura Recente e Análise Documental. Brasília/DF: UnB/TEDis, maio/2016 (mimeo).

YANNOULAS, S. C.; LENARDUZZI, Z. V. Texto Generador del Taller: entre la Cooperación y el Conflicto - Equipos Multiprofesionales-Multidisciplinares en el Ambiente Educacional. Brasília/DF: UnB/TEDis - UNER, jun./2016 (mimeo).

Texto recibido el 29 de agosto de 2016. Texto aprobado el 13 de diciembre de 2016. 
